\title{
Theory of optimum shapes in free-surface flows. Part 1. Optimum profile of sprayless planing surface
}

\author{
By T. YAO-TSU WU AND ARTHUR K. WHITNEY $\dagger$ \\ California Institute of Technology, Pasadena, California
}

(Received 8 September 1971 and in revised form 6 July 1972)

This paper attempts to determine the optimum profile of a two-dimensional plate that produces the maximum hydrodynamic lift while planing on a water surface, under the condition of no spray formation and no gravitational effect, the latter assumption serving as a good approximation for operations at large Froude numbers. The lift of the sprayless planing surface is maximized under the isoperimetric constraints of fixed chord length and fixed wetted arc-length of the plate. Consideration of the extremization yields, as the Euler equation, a pair of coupled nonlinear singular integral equations of the Cauchy type. These equations are subsequently linearized to facilitate further analysis. The analytical solution of the linearized problem has a branch-type singularity, in both pressure and flow angle, at the two ends of plate. In a special limit, this singularity changes its type, emerging into a logarithmic one, which is the weakest type possible. Guided by this analytic solution of the linearized problem, approximate solutions have been calculated for the nonlinear problem using the Rayleigh-Ritz method and the numerical results compared with the linearized theory.

\section{Introduction}

The problem of the planing surface has received much attention in the past as a device for producing hydrodynamic lift. Most of the early theoretical studies were based on linearized theory, taking into account the effect of gravity for the range of moderate to large Froude numbers, and assuming that the spray sheet at the leading edge of the plate is thrown backward in the upstream direction. The hydrodynamic drag on the planing surface therefore consists essentially of two components, one due to spray formation, and the other due to wave making, aside from the viscous skin-frictional drag which is generally small. A survey of the literature on the linear theory of planing surfaces has been given by Wehausen \& Laitone (1960). A crucial limitation of the linear theory, which seems to have escaped proper recognition, is that the plate draft (or the vertical distance of the plate from the undisturbed water surface) cannot be arbitrarily prescribed. Loss of this degree of freedom may be attributed to the pre-assigned direction of the spray sheet. This limitation was removed by Rispin (1967) and Wu (1967), who developed a nonlinear theory based on the singular perturbation method.

$\dagger$ Present address: Lockheed Palo Alto Research Laboratory, Lockheed Missiles and Space Co., Palo Alto, California. 
Of the previous investigations, Cumberbatch (1958) first explored an interesting case in which a planing surface operates, at a given Froude number, without spray formation, the so-called 'smooth entry' condition. This state of operation immediately opens up the possibility of further drag reduction by eliminating the spray, thereby improving the hydromechanical efficiency of the planing surface.

This paper seeks to determine the optimum profile of a two-dimensional plate, moving along the free surface of a uniform stream of water, without forming a spray sheet at the leading edge, such that for given chord length and wetted arclength of the plate, this profile will maximize the lift. For simplicity, the Froude number is assumed to be so large that the gravity effect may be neglected as the first approximation, or can be evaluated separately in a higher-order theory. The flow is further assumed to be inviscid and irrotational. Consequently, in the absence of the gravitational and viscous effects as woll as spray formation the planing surface will encounter no drag, leaving the lift as the only component of the hydrodynamic force.

Apart from its practical value in engineering applications, this problem was originally selected as one of the simplest in the general theory of optimum shapes involving free-surface flows, a theory which may have a far-reaching significance in its mathematical context. Generally speaking, in this class of variational problems, the functional subject to extremization contains unknown argument functions which are related to each other by integral equations, a consequence of the very nature of the mixed-type boundary problems. Further, for two-dimensional potential flows the integral equation is singular, of the Cauchy type. Thus, this situation is in sharp contrast to that in classical variational calculus, in which the unknown argument functions are related by differential equations. Consequently, the Euler equation which results from the consideration of extremization turns out to be, in general, a nonlinear, singular integral equation. Since the methods of solution for this class of equations are very limited, more powerful methods are very much desired. A preliminary mathematical study of this new class of variational problems has been carried out by the authors (Wu \& Whitney 1971). Following the same approach, the present problem will be investigated to. provide useful solutions of hydromechanical interest. It is hoped that this study will stimulate further interest in the development of the general theory, and, in turn, aid the resolution of numerous fluid mechanical problems of potential usefulness.

\section{The sprayless planing surface}

We consider the entire class of two-dimensional planing surfaces, with the plate profile so adjusted that the entry of water at the leading edge is 'smooth', i.e. without forming a spray sheet, as shown in figure 1. In the body frame: of reference the free-stream velocity is $U$, in the positive- $x$ direction, and the flow is assumed to be incompressible and irrotational. The Froude number $F r=U /(g l)^{\frac{1}{2}}$, based on the chord length $l$ and the gravitational constant $g$, is. taken to be so large that the effect of gravity may be neglected.

By a suitable translation and magnification, the complex potential $f=\phi+i \psi$, 

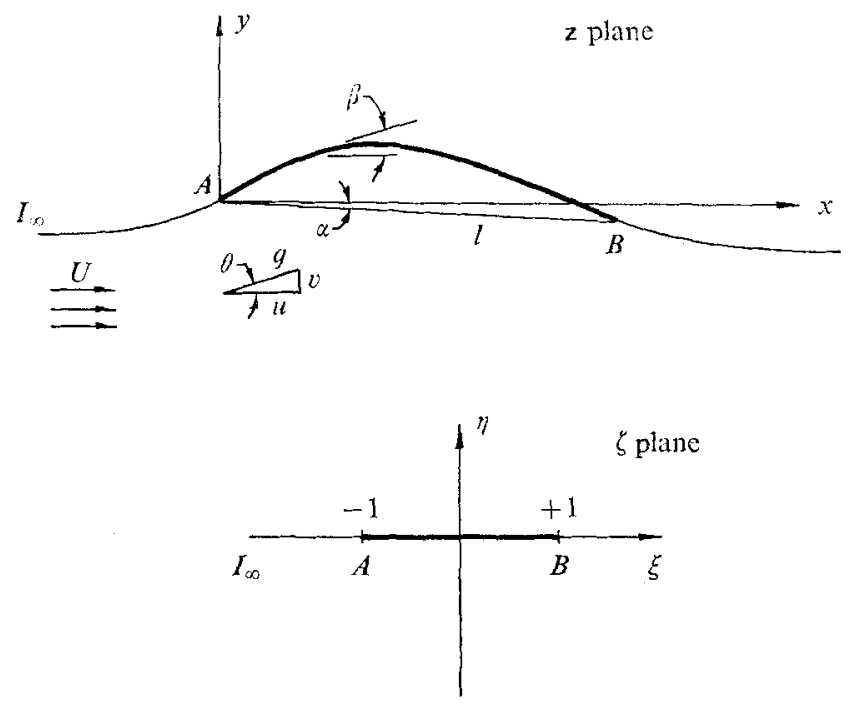

FIGURE 1. The physical and parametric planes.

$\phi$ being the velocity potential and $\psi$ the stream function, is mapped onto the lower half of a parametric $\zeta=\xi+i \eta$ plane by

$$
f=A U \zeta,
$$

$A$ being a real positive constant, with the plate mapped onto $\eta=0,-1<\xi<1$, and the free surface onto $\eta=0,|\xi|>1$. In terms of the physical plane $z=x+i y$ and the complex velocity

$$
w=d f / d z=u-i v=q e^{-i \theta}, \quad q=\left(u^{2}+v^{2}\right)^{\frac{1}{2}}, \quad \theta=\tan ^{-1}(v / u),
$$

or the logarithmic hodograph variable

$$
\omega=\log (U / w)=\tau+i \theta, \quad \tau=\log (U / q),
$$

the Bernoulli equation reads

$$
p-p_{0}=\frac{1}{2} \rho\left(U^{2}-q^{2}\right)=\frac{1}{2} \rho U^{2}\left(1-e^{-2 \tau}\right),
$$

$p$ being the pressure, $p_{0}$ its free-stream value, and $\rho$ the fluid density.

On the free surface $(\psi=0) p=p_{0}$, hence

$$
\tau^{-}(\xi)=\tau\left(\xi, 0^{-}\right)=0 \quad(|\xi|>1)
$$

On the plate, we denote the boundary value of $\omega(\zeta)$ by

$$
\omega^{-}(\xi-i 0)=\tau^{-}(\xi)+i \theta^{-}(\xi)=\Gamma(\xi)+i \beta(\xi) \quad(|\xi|<1) .
$$

As for the boundary condition on the plate, the simplest approach is to consider the 'inverse problem' by prescribing either $\Gamma(\xi)$, or $\beta(\xi)$, as a known function of $\xi$, together with certain conditions to be specified below. When $\Gamma(\xi)$ is prescribed, it is required to be Hölder-continuous $\dagger$, non-negative (to ensure that the pressure

$\dagger \Gamma(\xi)$ is said to be Hölder-continuous on $[-1,1]$ if for any two points $\xi_{1}, \xi_{2}$ on $[-1,1]$, $\left|\Gamma\left(\xi_{1}\right)-\Gamma\left(\xi_{2}\right)\right|<B\left|\xi_{1}-\xi_{2}\right| \mu$, with the Hölder constant $B>0$, and the Hölder index $\mu$ satisfying $0<\mu \leqslant 1$. 
on the plate is nowhere less than $\left.p_{0}\right)$, and $\tau^{-}(\xi)$ is required to be continuous across the two ends of the plate at $\xi= \pm 1$, i.e.

$$
\begin{gathered}
\tau-(\xi)=\Gamma(\xi) \geqslant 0 \quad(|\xi| \leqslant 1), \\
\Gamma(1)=\Gamma(-1)=0
\end{gathered}
$$

Under the present assumptions, the free-stream condition is simply

$$
\omega \rightarrow 0 \quad \text { as } \quad|\zeta| \rightarrow \infty, \quad(\eta \leqslant 0) .
$$

The solution of the Dirichlet problem prescribed by (5), (7) and (9) is

$$
\omega(\zeta)=\frac{i}{\pi} \int_{-1}^{1} \frac{\Gamma(t) d t}{t-\zeta} \quad(\eta=\operatorname{Im} \zeta \leqslant 0)
$$

As $\zeta$ approaches an arbitrary point $\zeta=\xi-i 0$ on the plate, use of the Plemelj formula (cf. e.g. Muskhelishvili 1953) shows that the real part of (10) reduces to an identity, and its imaginary part gives

$$
\beta(\xi)=\frac{1}{\pi} \oint_{-1}^{1} \frac{\Gamma(t) d t}{t-\xi} \equiv H_{\xi}[\Gamma(t)] \quad(|\xi|<1),
$$

in which the symbol $C$ over the integral signifies its Cauchy principal value, and the symbol $H_{\xi}[\Gamma]$ denotes the finite Hilbert transform of $\Gamma$ on $[-1,1]$. It is noted that if $\Gamma(\xi)$ is Hölder-continuous on $[-1,1]$ and if the end conditions (8) are satisfied, then $\beta(\xi)$, given by (11), is also Hölder-continuous on $[-1,1]$ (see Muskhelishvili 1953, §19, p. 29).

If, instead of $\tau^{-}(\xi), \theta^{-}(\xi)=\beta(\xi)$ is prescribed for $|\xi|<1$, one may either solve this Riemann-Hilbert problem directly, or regard (11) as an integral equation for $\Gamma(\xi)$. The solution for $\Gamma(\xi)$ satisfying conditions (8) and (9) is found to be

$$
\Gamma(\xi)=-\frac{1}{\pi}\left(1-\xi^{2}\right)^{\frac{1}{2}} \oint_{-1}^{1} \frac{\beta(t) d t}{\left(1-t^{2}\right)^{\frac{1}{2}}(t-\xi)} \quad(|\xi|<1)
$$

provided $\beta(\xi)$ further satisfies the orthogonality condition

$$
\int_{-1}^{1} \beta(\xi)\left(1-\xi^{2}\right)^{-\frac{1}{2}} d \xi=0
$$

In the above and in the sequel, the function $\left(\zeta^{2}-1\right)^{\frac{1}{2}}$ is defined to be one-valued in the entire complex $\zeta$ plane, cut from $\zeta=-1$ to 1 along the real $\zeta$ axis, so that $\left(\zeta^{2}-1\right)^{\frac{1}{2}} \rightarrow \zeta$ as $|\zeta| \rightarrow \infty$ for all arg $\zeta$. Thus, $\left(\zeta^{2}-1\right)^{\frac{1}{2}} \rightarrow \pm i\left(1-\xi^{2}\right)^{\frac{1}{2}}$ as $\zeta \rightarrow \xi \pm i 0$, $|\xi|<1$. It may be remarked here that the system (8) and (11) is equivalent to the system (12) and (13). Furthermore, by virtue of condition (5), $\omega(\zeta)$ can be continued analytically into the upper half $\zeta$ plane by

$$
\omega(\bar{\zeta})=-\overline{\omega(\zeta)}
$$

The physical plane is obtained by integration of $w=d f / d z$,

$$
z(\zeta)=A U \int_{-1}^{\zeta} d \zeta / w(\zeta)=A \int_{-1}^{\zeta} e^{\omega(\zeta)} d \zeta
$$


with $z(-1)=0$. The chord of the plate $l$ and its angle of incidence to the free stream, $\alpha$ (positive in the clockwise sense), are given by

$$
\begin{gathered}
l=A \int_{-1}^{1} e^{\Gamma(\xi)} \cos [\beta(\xi)+\alpha] d \xi \\
\int_{-1}^{1} e^{\Gamma(\xi)} \sin [\beta(\xi)+\alpha] d \xi=0
\end{gathered}
$$

Equation (16) determines the factor $A$ in the transformation (1), and (17) states that the angle of attack $\alpha$ is referred to the chord of the plate. Finally, the total arc-length $S$ of the plate is

$$
S=A \int_{-1}^{1} e^{\Gamma(\xi)} d \xi
$$

The total force $F=D+i L$, consisting of the drag $D$ and lift $L$ acting on the plate, can be determined from

$$
\begin{aligned}
F & =i \int_{A}^{B}\left(p-p_{0}\right) d z=\frac{1}{2} i \rho \int_{-1}^{1}\left(U^{2}-w \bar{w}\right) \frac{1}{w} \frac{d f}{d \zeta} d \zeta \\
& =\frac{1}{2} i \rho U^{2} A \int_{-1}^{1}\left[e^{\omega(\zeta)}-e^{-\overline{\omega(\zeta)}}\right] d \zeta=\frac{1}{2} i \rho U^{2} A \oint e^{\omega(\zeta)} d \zeta
\end{aligned}
$$

where the contour of the last integral encircles the plate counter-clockwise in the $\zeta$ plane upon using the analytic continuation (14). By expanding $\omega(\zeta)$, given by (10), for large $|\zeta|$, we obtain, by the theory of residues,

$$
L=\rho U^{2} A \int_{-1}^{1} \Gamma(\xi) d \xi, \quad D=0 .
$$

Thus, the drag $D=0$, as should be expected since there is no mechanism for producing drag, by wave making or other means, in this idealized case.

\section{The optimum shape problem}

We now consider the optimum shape problem. In the class of functions $\Gamma(\xi)$ which are supposed to be Hölder-continuous on $[-1,1]$, to satisfy the inequality condition (7) and the homogeneous end conditions (8), find the extremal arc $\Gamma_{0}(\xi)$ and its conjugate $\beta_{0}(\xi)$, mutually related by (11), which maximize the lift $L$ under the isoperimetric constraints of fixed chord $l$ and total arc-length $S$.

In what follows we shall assume that the extremal arc has the property $\alpha=0$ (zero incidence of the chord) and the symmetry

$$
\Gamma(-\xi)=\Gamma(\xi), \quad \beta(-\xi)=-\beta(\xi)
$$

The fact that the solution, if unique, must have this property may be seen by observing that the extremal arc will remain extremal when the flow direction is reversed. Under this condition, (17) is then automatically satisfied.

The problem of maximizing the lift $L=\rho U^{2} L^{*}$ (see (20)) under the isoperimetric constraints of fixed chord $l$ (see (16), now with $\alpha=0$ ) and given arelength $S$ (see (18)) is equivalent to that of finding the pair of extremal arcs $\Gamma(\xi)$, 
$\beta(\xi)$, which, while satisfying (7), (8) and (11), will also minimize the new functional

$$
\begin{gathered}
I[\Gamma, \beta ; A]=\lambda_{1} l+\lambda_{2} S-L^{*}=A \int_{-1}^{1} f\left(\Gamma(\xi), \beta(\xi) ; \lambda_{1}, \lambda_{2}\right) d \xi \\
f\left(\Gamma, \beta ; \lambda_{1}, \lambda_{2}\right)=\lambda_{1} e^{\Gamma(\xi)} \cos \beta(\xi)+\lambda_{2} e^{\Gamma(\xi)}-\Gamma(\xi) \quad(|\xi|<1) .
\end{gathered}
$$

Here, $\lambda_{1}, \lambda_{2}$ are undetermined multipliers, and the negative sign given to $L^{*}$ makes minimization of $I[\Gamma, \beta ; A]$ correspond to maximization of $L^{*}$. It is necessary to include the coefficient $A$ in the arguments of $I$, since for fixed $l$ and $S, A$ is a functional of $\Gamma$ and $\beta$.

The general variational problem of this kind has been discussed recently by Wu \& Whitney (1971). For the problem at hand, the method of solution will require some modifications of the same approach, and will be made self-contained here. Let the set $[\Gamma(\xi), \beta(\xi) ; A]$ denote the optimal solution and let $\left[\Gamma_{1}(\xi), \beta_{1}(\xi)\right.$; $\left.A_{1}\right]$ be an arbitrary neighbouring admissible set, which, by definition, satisfies (11), conditions (7) and (8), and the Hölder-continuity condition. The differences $\delta \Gamma=\Gamma_{1}(\xi)-\Gamma(\xi), \delta \beta=\beta_{1}(\xi)-\beta(\xi), \delta A=A_{1}-A$ form a set of arbitrarily small variations. Suppose $\delta \Gamma(\xi)$ is taken to be a small arbitrary function of $\xi$; then, since both $[\Gamma, \beta]$ and $\left[\Gamma_{1}, \beta_{1}\right]$ satisfy $(11), \delta \beta(\xi)$ is given by the Hilbert transform of $\delta \Gamma$,

$$
\delta \beta(\xi)=H_{\xi}[\delta \Gamma] \quad(|\xi|<1) .
$$

The variation $\delta A$, however, is arbitrary.

The variation of the functional $I$ corresponding to $[\delta \Gamma, \delta \beta, \delta A]$ is

$$
\Delta I=(A+\delta A) \int_{-1}^{1} f(\Gamma+\delta \Gamma, \beta+\delta \beta) d \xi-A \int_{-1}^{1} f(\Gamma, \beta) d \xi
$$

Expansion of the above expression for sufficiently small $|\delta \Gamma|,|\delta \beta|,|\delta A|$ yields

$$
\Delta I=\delta I+\frac{1}{2 !} \delta^{2} I+\frac{1}{3 !} \delta^{3} I+\ldots
$$

where the first variation $\delta I$ and the second variation $\delta^{2} I$ are

$$
\begin{gathered}
\delta I=(\delta A) \int f(\Gamma, \beta) d \xi+A \int\left(f_{\Gamma} \delta \Gamma+f_{\beta} \delta \beta\right) d \xi \\
\delta^{2} I=2(\delta A) \int\left(f_{\Gamma} \delta \Gamma+f_{\beta} \delta \beta\right) d \xi+A \int\left[f_{\Gamma \Gamma}(\delta \Gamma)^{2}+2 f_{\Gamma \beta} \delta \Gamma \delta \beta+f_{\beta \beta}(\delta \beta)^{2}\right] d \xi
\end{gathered}
$$

in which the subscripts denote partial differentiations, and all integrals are from $\xi=-1$ to 1 . For $I[\Gamma, \beta ; A]$ to be a minimum, we must have $\delta I=0$ and $\delta^{2} I>0$ for arbitrary $\delta \Gamma$ and $\delta A$. From $\delta I=0$ it then follows that the two integrals in the expression for $\delta I$ must vanish separately. The first integral vanishes if, by $(22 a)$,

$$
\begin{gathered}
L^{*}=\lambda_{1} l+\lambda_{2} S, \\
\text { or explicitly, } \quad \int_{-1}^{1} \Gamma(\xi) d \xi=\int_{-1}^{1} e^{\Gamma(\xi)}\left[\lambda_{1} \cos \beta(\xi)+\lambda_{2}\right] d \xi
\end{gathered}
$$

which provides one condition for the constant multipliers $\lambda_{1}, \lambda_{2}$. (Note that the positive coefficient $A$ drops out in (24b).) For the second integral, we substitute 
(23) for $\delta \beta$, then interchange the order of integration, which is permissible under certain conditions (see e.g. Tricomi 1957, $\$ 4.3$ ), giving

$$
\int_{-1}^{1}\left(f_{\Gamma} \delta \Gamma+f_{\beta} \delta \beta\right) d \xi=\int_{-1}^{1}\left(f_{\Gamma}-H_{\xi}\left[f_{\beta}\right]\right) \delta \Gamma(\xi) d \xi=0 .
$$

Since $\delta \Gamma(\xi)$ is arbitrary, we obtain the nonlinear singular integral equation of the Cauchy type,

where, by $(22 b)$,

$$
f_{\Gamma}(\Gamma(\xi), \beta(\xi))=H_{\xi}\left[f_{\beta}\right]=\frac{1}{\pi} \oint_{-1}^{1} \frac{f_{\beta}(\Gamma(t), \beta(t))}{t-\xi} d t \quad(|\xi|<1),
$$

$$
\frac{\partial f}{\partial \Gamma}=e^{\Gamma(\xi)}\left[\lambda_{1} \cos \beta(\xi)+\lambda_{2}\right]-1, \quad \frac{\partial f}{\partial \beta}=-\lambda_{1} e^{\Gamma(\xi)} \sin \beta(\xi)
$$

For the extremal solution, (26) is to be solved together with (11), as a pair of singular integral equations for $\Gamma(\xi)$ and $\beta(\xi)$, subject to the homogeneous end conditions (8) and the inequality condition (7). The extremal solution, $\Gamma\left(\xi ; \lambda_{1}, \lambda_{2}\right)$ and $\beta\left(\xi ; \lambda_{1}, \lambda_{2}\right)$, when determined in this manner, will involve the two constant multipliers $\lambda_{1}$ and $\lambda_{2}$, which can be determined, most conveniently, by applying condition (24), and by giving a specified ratio of the arc-length $S$ to the chord $l$, say

or, by using (16), (18),

$$
S / l=1+\kappa \quad(\kappa>0)
$$

$$
\int_{-1}^{1} e^{\Gamma(\xi)} d \xi=(1+\kappa) \int_{-1}^{1} e^{\Gamma(\xi)} \cos \beta(\xi) d \xi
$$

Since the coefficient $A$ does not appear in either of the isoperimetric conditions (24) and (27), the problem of determining the unknown $A$ is curtailed altogether. It is nevertheless necessary to consider the variation $\delta A$ to obtain (24). Finally, the optimum lift coefficient, upon using (24a) and (27a), can be expressed as

$$
C_{L}=L /\left(\frac{1}{2} \rho U^{2} l\right)=2 L^{*} / l=2 \lambda_{1}+2 \lambda_{2}(1+\kappa)
$$

This optimum lift coefficient will be a maximum if the second variation of $I$ satisfies the inequality condition $\delta^{2} I>0$, which becomes

$$
\int_{-1}^{1}\left[f_{\Gamma \Gamma}(\delta \Gamma)^{2}+2 f_{\Gamma \beta} \delta \Gamma \delta \beta+f_{\beta \beta}(\delta \beta)^{2}\right] d \xi>0
$$

upon incorporating (25) and noting that $A>0$. A necessary condition for the above inequality to hold was found by Whitney (1969) to be

$$
f_{\Gamma \Gamma}(\Gamma(\xi), \beta(\xi))+f_{\beta \beta}(\Gamma(\xi), \beta(\xi))>0 \quad(-1<\xi<1) .
$$

The procedure for obtaining this result is first to substitute (23) for the $\delta \beta$ 's in the integrand, interchange the order of integration by the Poincaré-Bertrand formula, and then to consider a special choice of $\delta \Gamma(\xi)$ which vanishes everywhere except on an infinitesimal stretch in $(-1<\xi<1)$. For the present problem, with $f$ given by $(22 b),(29)$ gives

$$
f_{\Gamma \Gamma}+f_{\beta \beta}=\lambda_{2} e^{\Gamma(\xi)}>0, \quad \text { or simply } \lambda_{2}>0,
$$


since the optimum solution $\Gamma$ is real. It may be remarked here that $C_{L}$ in (28) will be a minimum when $\lambda_{2}<0$. Condition (30) also shows the importance of including the arc-length $S$ as a constraint; otherwise, the necessary condition (29) cannot be satisfied, and the consideration of optimality must necessarily proceed to higher-order variations of the functional $I$, to say the least.

The exact solution of this problem is exceedingly difficult for several reasons. First of all, (11) and (26) are a system of nonlinear singular integral equations, with a Cauchy kernel, which have no known general method of solution. Second, it appears to be very difficult to incorporate automatically the inequality condition $(7), \Gamma(\xi) \geqslant 0$ for $|\xi|<1$, into the analysis, the only alternative being to verify its validity if and when all possible solutions for $\Gamma$ have been obtained. Furthermore, there is no assurance that the homogeneous end conditions (8), $\Gamma(1)=\Gamma(-1)=0$, can always be satisfied. Finally, even when the solution of $\Gamma$ satisfying all these conditions can be obtained, the determination of the multipliers $\lambda_{1}, \lambda_{2}$ from (24), (27) will involve equations which are highly transcendental. The foregoing observations should indicate that any plausible method of solution by numerical iterations would most likely meet great resistance.

However, important information about the solution can be obtained from the corresponding linearized theory, which we proceed to consider in $§ 4$.

\section{The linearized integral equation, for $(S-l) / l \ll 1$}

A linearized theory may be developed to provide a valid first-order solution to $\Gamma(\xi)$ and $\beta(\xi)$ when the arc-length $S$ is only slightly greater than the chord $l$, or

$$
S / l=1+\kappa, \quad 0<\kappa \ll 1 .
$$

In this limit, $\Gamma(\xi)$ and $\beta(\xi)$ are expected to be almost everywhere small on $(-1<\xi<1)$, except possibly near the endpoints $\xi= \pm 1$. Thus, upon expanding $f_{\Gamma}$ and $f_{\beta}$ for small $|\Gamma|$ and $|\beta|$, and keeping only the linear terms, (26) reduces to

where

$$
a \Gamma(\xi)=c H_{\xi}[\beta(t)]+(1-a) \quad(|\xi|<1),
$$

The linear system of singular integral equations (32) and (11) belongs to a class previously investigated by Wu \& Whitney (1971); it is a system that can be uncoupled to yield a set of singular integral equations of the Carleman type and then solved by known methods. Without going through the detailed analysis, we give below the final solution, which can be readily verified. The solution has two branches according as the coefficient

$$
\sigma=c / a=-\lambda_{1} /\left(\lambda_{1}+\lambda_{2}\right)>0 \text { or }<0 .
$$

(i) First case $\sigma>0$

The ranges of $\lambda_{1}$ and $\lambda_{2}$ in this case are either

$$
\lambda_{2}>-\lambda_{1}>0 \quad \text { or } \quad \lambda_{2}<-\lambda_{1}<0
$$


the first, according to the necessary condition (30), corresponds to the maximum lift, whereas the second corresponds to the minimum lift. In terms of the new parameters $\gamma$ and $\mu$, defined by

$$
\gamma=(1-a) / a \quad \text { and } \quad \tan \mu \pi=\sigma^{\frac{1}{2}} \quad\left(0<\mu<\frac{1}{2}\right)
$$

the solution is given by

$$
\begin{gathered}
\Gamma(\xi)=\frac{\gamma}{2} \cos \mu \pi\left[\left(\frac{1-\xi}{1+\xi}\right)^{\mu}+\left(\frac{1-\xi}{1+\xi}\right)^{-\mu}\right] \\
\beta(\xi)=\frac{\gamma}{2} \cos \mu \pi \cot \mu \pi\left[\left(\frac{1-\xi}{1+\xi}\right)^{\mu}-\left(\frac{1-\xi}{1+\xi}\right)^{-\mu}\right] .
\end{gathered}
$$

This solution is readily verified by making use of the formula

$$
H_{\xi}\left[\left(\frac{1-\xi}{1+\xi}\right)^{ \pm \mu}\right]= \pm(\cot \mu \pi)\left(\frac{1-\xi}{1+\xi}\right)^{ \pm \mu} \mp \csc \mu \pi \quad(|\xi|<1)
$$

which can be derived directly by contour integration of $(t-1)^{\mu}(t+1)^{-\mu}(t-\xi)^{-1}$ in the complex $t$ plane (or see Tricomi 1957, p. 181). We note that the above solution satisfies the inequality condition $(7)$, i.e. $\Gamma(\xi)>0(|\xi|<1)$, but is singular, with a branch-type singularity, at the endpoints $\xi= \pm 1$, and thus fails to satisfy the homogeneous end condition (8). Whether these singularities can be removed by including the nonlinear terms remains to be seen.

We now determine the Lagrange multipliers $\lambda_{1}, \lambda_{2}$ from (24) and (27). To be consistent within the framework of the linearized theory, all the nonlinear functions in the integrands of $(24 b)$ and $(27 b)$ will be expanded for small $|\Gamma|$ and $|\beta|$ up to the quadratic terms, because the linear integral equation (32) actually follows from (26) by expanding the fundamental function $f(\Gamma, \beta)$ up to the terms with $\Gamma^{2}, \Gamma \beta$, and $\beta^{2}$. Thus, $(24 b)$ and $(27 b)$ reduce, after some rearrangement, respectively to

$$
\left.\begin{array}{l}
2-\gamma \int_{-1}^{1} \Gamma(\xi) d \xi+\frac{1}{2} \int_{-1}^{1} \Gamma^{2}(\xi) d \xi+\frac{1}{2} \sigma \int_{-1}^{1} \beta^{2}(\xi) d \xi=0 \\
2+\int_{-1}^{1} \Gamma(\xi) d \xi+\frac{1}{2} \int_{-1}^{1} \Gamma^{2}(\xi) d \xi-\frac{1+\kappa}{2 \kappa} \int_{-1}^{1} \beta^{2}(\xi) d \xi=0
\end{array}\right\}
$$

where $\sigma$ and $\gamma$ are defined by (33) and (35). The difference between the above two equations gives

$$
\int_{-1}^{1} \Gamma(\xi) d \xi=\frac{1}{2}\left(\frac{\lambda_{1}}{\kappa}+\lambda_{2} \frac{1+\kappa}{\kappa}\right) \int_{-1}^{1} \beta^{2}(\xi) d \xi,
$$

which can be used with (37) as two isoperimetric conditions. The integrals involved in (37), (38) are easily found from the solution (36) as

$$
\begin{aligned}
& \int_{-1}^{1} \Gamma(\xi) d \xi=2 \gamma \mu \pi \sigma^{-\frac{1}{2}} \\
& \int_{-1}^{1} \Gamma^{2}(\xi) d \xi=\gamma^{2}\left[\mu \pi \sigma^{-\frac{1}{2}}+(1+\sigma)^{-1}\right] \\
& \int_{-1}^{1} \beta^{2}(\xi) d \xi=\gamma^{2} \sigma^{-1}\left[\mu \pi \sigma^{-\frac{1}{2}}-(1+\sigma)^{-1}\right] .
\end{aligned}
$$




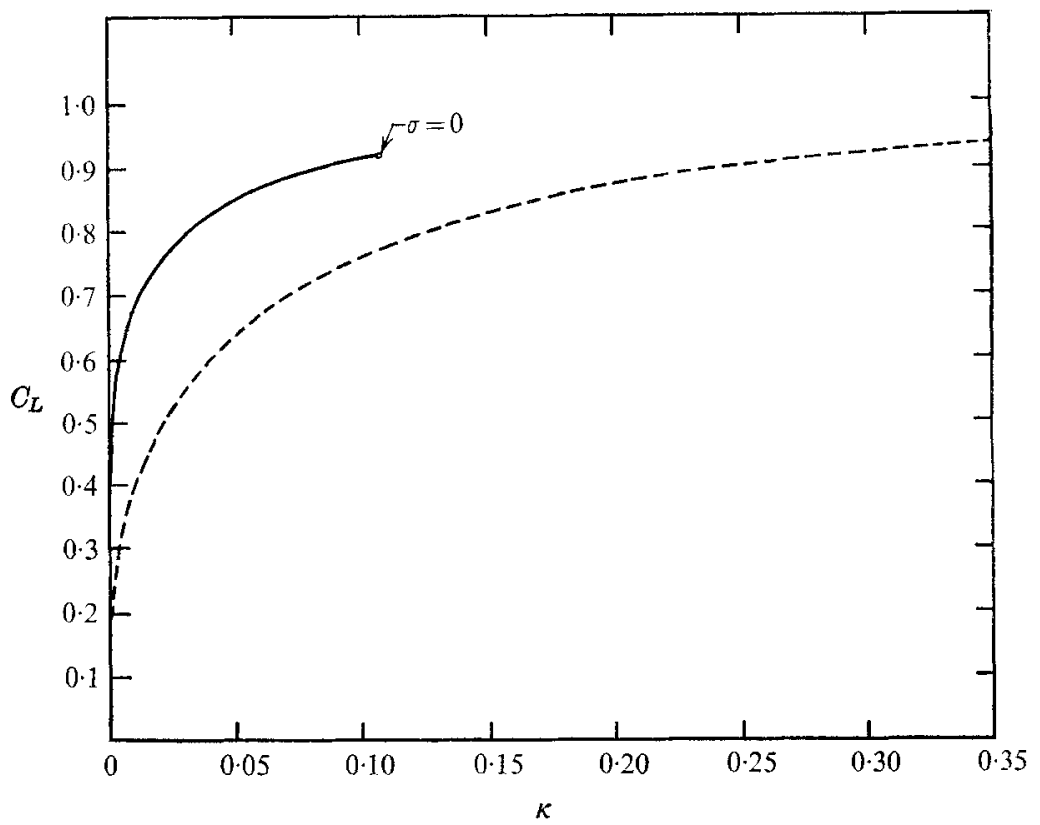

FigURe 2. Optimum lift coefficient $C_{L}$ against $\kappa=S / l-1$ for the linearized theory ( $\longrightarrow$ ) and the two-term nonlinear Fourier series expansion (-- - - ).

Substitution of these integrals in (37) and (38) yields

$$
\begin{gathered}
\gamma^{2} \mu \pi=2 \sigma^{\frac{1}{2}} \\
4 \sigma=\gamma\left(\frac{\lambda_{1}}{\kappa}+\lambda_{2} \frac{1+\kappa}{\kappa}\right)\left[1-\frac{\sigma^{\frac{1}{2}}}{(1+\sigma) \mu \pi}\right] .
\end{gathered}
$$

These two equations determine $\lambda_{1}$ and $\lambda_{2}$ in terms of $\kappa$, or more conveniently, in terms of $\sigma$ or $\mu$. From (33), (35), and (39), we have

$$
\lambda_{1}=-\sigma /(1+\gamma), \quad \lambda_{2}=(1+\sigma) /(1+\gamma),
$$

where

$$
\gamma= \pm\left[2 \sigma^{\frac{1}{2}} / \tan ^{-1}\left(\sigma^{\frac{1}{2}}\right)\right]^{\frac{1}{2}}= \pm[(2 \tan \mu \pi) / \mu \pi]^{\frac{1}{2}}
$$

and the + sign is for maximum lift, the - sign for minimum lift. Substituting these expressions for $\lambda_{1}, \lambda_{2}$, and $\gamma$, into (28) and (40), the optimal lift coefficient $C_{L}$ as a function of $\kappa$ is given parametrically by

$$
\begin{gathered}
C_{L}= \pm 4[2 \mu \pi \cot \mu \pi]^{\frac{1}{2}} / l(\mu), \\
\kappa=\cot ^{2} \mu \pi\left[1-\frac{\sin 2 \mu \pi}{2 \mu \pi}\right] / l(\mu),
\end{gathered}
$$

where

$$
l(\mu)=4-\csc ^{2} \mu \pi\left(1-\frac{\sin 2 \mu \pi}{2 \mu \pi}\right) \pm(8 \mu \pi \cot \mu \pi)^{\frac{1}{2}} .
$$

The maximum lift coefficient $(+\operatorname{sign}$ in (41)) as a function of $\kappa$ is shown in figure 2. The following limiting cases are of special interest. 
(ia) $\kappa \rightarrow 0+(\sigma \rightarrow+\infty)$. By reducing $\kappa$ to zero, the plate is constrained to be nearly stretched straight, implying that $\Gamma$ and $\beta \simeq O(\Gamma)$ are both small. Setting $\sigma=\epsilon^{-2} \gg 1$, we deduce from $(39)$ and $(41 b, c)$ that

and

$$
\mu=\frac{1}{2}-\epsilon / \pi+O\left(\epsilon^{3}\right), \quad \gamma= \pm 2(\epsilon \pi)^{-\frac{1}{2}}\left[1+\epsilon / \pi+O\left(\epsilon^{2}\right)\right],
$$

Finally, the optimal lift coefficient, by $(41 a)$, is

$$
C_{L}= \pm \frac{4}{3}\left(3 \pi^{2} \kappa\right)^{\frac{1}{4}}\left[1 \mp \frac{1}{2}\left(3 \pi^{2} \kappa\right)^{\frac{1}{4}}+O\left(\kappa^{\frac{1}{2}}\right)\right] .
$$

This expansion of the optimum solution, valid for very small $\kappa$ (such as $<0.005$ ) shows that as soon as $\kappa$ increases from zero, the plate starts to bend more near the two ends and carries most of the lift there, since, by $(36)$ and (42), both $\Gamma$ and $\beta$ have square-root singularities at $\xi= \pm 1$. As the arc-length $S$ further increases (for fixed chord $l$ ), $\kappa$ and $\epsilon$ become greater, $\mu$ smaller, and the singularities at the ends then become weaker. The rate of increase of the optimum lift with respect to increasing $\kappa, d C_{L} / d \kappa$, behaves like $\kappa^{-\frac{3}{4}}$ as $\kappa \rightarrow 0$.

(ib) $0<\sigma \ll 1$. Another interesting limit is as $\sigma \rightarrow 0+$, in which case we find

$$
\begin{gathered}
\mu=(1 / \pi) \sigma^{\frac{1}{2}}\left[1-\frac{1}{3} \sigma+O\left(\sigma^{2}\right)\right], \quad \gamma= \pm \sqrt{ } 2\left[1+\frac{1}{6} \sigma+O\left(\sigma^{2}\right)\right], \\
\kappa=\frac{1}{7}(5 \mp 3 \sqrt{ } 2)\left[1-(3 \sigma / 10)(4 \mp \sqrt{ } 2)+O\left(\sigma^{2}\right)\right] .
\end{gathered}
$$

Here, the upper (or lower) sign is for the maximum (or minimum) lift coefficient, which is obtained from $(41 a, c)$ as

$$
C_{L}= \pm \frac{6}{7}(5 \sqrt{ } 2 \mp 6)\left[1-\frac{\sigma}{10}(5 \mp 3 \sqrt{ } 2)+O\left(\sigma^{2}\right)\right] .
$$

In the limit as $\sigma \rightarrow 0+$, the maximum lift coefficient

$$
C_{L \max } \simeq 0.918(1-0.76 \sigma) \text { at } \kappa=0.1082(1-0.776 \sigma)
$$

appears to be quite high, particularly for such small $\kappa$. On the other hand, it seems rather questionable whether the minimum lift coefficient

$$
C_{L \min } \simeq-11 \cdot 20(1-0.924 \sigma) \text { at } \kappa=1 \cdot 320(1-1 \cdot 624 \sigma)
$$

might be physically realizable because the low pressure underneath the plate would most likely cause air to ventilate the entire lower surface.

The corresponding solution of $\Gamma$ and $\beta$ can be immediately deduced from (36) as

$$
\begin{gathered}
\Gamma(\xi)= \pm \sqrt{ } 2\left\{1+\frac{\sigma}{2}\left[\frac{1}{\pi^{2}}\left(\log \frac{1-\xi}{1+\xi}\right)^{2}-\frac{2}{3}\right]+O\left(\sigma^{2}\right)\right\} \\
\beta(\xi)= \pm \frac{\sqrt{ } 2}{\pi} \log \left(\frac{1-\xi}{1+\xi}\right)\left\{1+\frac{\sigma}{6}\left[\frac{1}{\pi^{2}}\left(\log \frac{1-\xi}{1+\xi}\right)^{2}-1\right]+O\left(\sigma^{2}\right)\right\} .
\end{gathered}
$$

Thus, in this limit, the flow angle $\beta(\xi)$ has a logarithmic singularity at the endpoints $\xi= \pm 1$, which are of the weakest type in this linearized theory. This particular profile of the plate results in a constant pressure distribution over the plate except for a higher-order logarithmio singularity at the two edges. The overall features of the solution in the present limiting case therefore suggest that this is the most favourable optimum state arrived at for small variations of parameter $\kappa$. 
(ii) Second case $-1<\sigma<0$

The ranges of $\lambda_{1}$ and $\lambda_{2}$ now become either

$$
\lambda_{1}>0, \lambda_{2}>0 \text { or } \lambda_{1}<0, \lambda_{2}<0,
$$

corresponding, respectively, to the maximum and minimum lift. In terms of the parameter $v$ defined by

$$
\tanh \nu \pi=(-\sigma)^{\frac{1}{2}} \quad(\nu>0),
$$

the solution of (11) and (32) for this case is found as

$$
\begin{gathered}
\Gamma(\xi)=\gamma \cosh \nu \pi \cos \left(\nu \log \frac{1-\xi}{1+\xi}\right), \\
\beta(\xi)=\gamma \cosh \nu \pi \operatorname{coth} \nu \pi \sin \left(\nu \log \frac{1-\xi}{1+\xi}\right),
\end{gathered}
$$

where the coefficient $\gamma$ is given by (35). This solution can be immediately verified by making use of the formulae

$$
\begin{aligned}
& H_{\xi}\left[\sin \left(\nu \log \frac{1-t}{1+t}\right)\right]=\operatorname{csch} \nu \pi-\operatorname{coth} \nu \pi \cos \left(\nu \log \frac{1-\xi}{1+\xi}\right) \\
& H_{\xi}\left[\cos \left(\nu \log \frac{1-t}{1+t}\right)\right]=\operatorname{coth} \nu \pi \sin \left(\nu \log \frac{1-\xi}{1+\xi}\right) \quad(|\xi|<1) .
\end{aligned}
$$

The above inversion formulae can be derived by a contour integration of

$$
(t-1)^{i v}(t+1)^{-i v}(t-\xi)^{-1}
$$

encircling the real axis from $t=-1$ to 1 in the complex $t$ plane.

Although both of the above $\Gamma$ and $\beta$ remain bounded in $-1 \leqslant \xi \leqslant 1$, they nevertheless oscillate infinitely fast as the endpoints $\xi= \pm 1$ are approached, and hence do not satisfy the inequality condition (7), as is required on physical grounds. For this reason the above solution is regarded as void of any physical significance, and hence will not be further pursued here, although it also tends to the limiting solution (47), (48) as $\nu \rightarrow 0$. However, to include this case in our discussion may usefully serve to point out that when a numerical iteration method is employed, particularly for small values of $\sigma$, the iterated solutions may oscillate between the two cases (ib) and (ii), and the success of such procedure may be hindered by lack of convergence.

\section{Discretized Fourier series expansions: the Rayleigh-Ritz method}

We next consider a method for obtaining approximate solutions to the optimum shape problem by expanding $\Gamma(\xi)$ and $\beta(\xi)$ in finite Fourier series with the coefficients so chosen that the lift is maximized under the present isoperimetric constraints of fixed chord and fixed wetted arc-length.

Let the expansion for $\Gamma(\xi)$ be given by

$$
\Gamma(\xi)=\sum_{n=1}^{N} a_{n} \sin (2 n-1) \theta
$$


where $\xi=\cos \theta(0 \leqslant \theta \leqslant \pi)$ and the $a_{n}$ 's are arbitrary real constants. This choice for $\Gamma$ automatically satisfies the end conditions (8) and the symmetry property (21). From (11), $\beta(\xi)$ is given by

which follows from the identity

$$
\beta(\xi)=-\sum_{n=1}^{N} a_{n} \cos (2 n-1) \theta
$$

$$
\oint_{0}^{\pi} \frac{\sin m \phi \sin \phi}{\cos \phi-\cos \theta} d \phi=-\pi \cos m \theta
$$

The problem of maximizing the lift for fixed arc-length and chord is equivalent to minimizing the functional $I$ in (22), which, by (55) and (56), may now be considered as an ordinary function of the coefficients $\left\{a_{n}\right\}$ and the factor $A$. To minimize $I$ we set the partial derivatives of $I$ with respect to $A$ and each of the $a_{n}$ 's to zero, giving (since $A$ does not vanish)

$$
\begin{gathered}
\frac{\partial I}{\partial A}=\frac{I}{A}=\int_{0}^{\pi} e^{\Gamma}\left[\lambda_{1} \cos \beta+\lambda_{2}\right] \sin \theta d \theta-\frac{1}{2} \pi a_{1}=0 \\
\frac{1}{A} \frac{\partial I}{\partial a_{n}}=\int_{0}^{\pi} e^{\Gamma}\left\{\lambda_{1} \sin [\beta+(2 n-1) \theta]+\lambda_{2} \sin (2 n-1) \theta\right\} \sin \theta d \theta-\frac{1}{2} \pi \delta_{n 1}=0 \\
(n=1,2, \ldots, N) .
\end{gathered}
$$

The $(N+1)$-equations above, in which $\Gamma$ and $\beta$ are given by (55) and (56), together with (27) in which $\kappa$ is given, determine the $N$ Fourier coefficients $\left\{a_{n}\right\}$ and the two Lagrange multipliers $\lambda_{1}, \lambda_{2}$. Finally, the lift coefficient is given by (28). Since (57) and (58) are transcendental in the unknown Fourier coefficients the solutions must be found numerically.

In order to illustrate this method we consider the case $N=2$. Equations (58) read as follows:

$$
\begin{aligned}
& \lambda_{1} \int_{0}^{\pi} e^{\Gamma} \sin (\beta+\theta) \sin \theta d \theta+\lambda_{2} \int_{0}^{\pi} e^{\Gamma} \sin ^{2} \theta d \theta=\frac{1}{2} \pi \\
& \lambda_{1} \int_{0}^{\pi} e^{\Gamma} \sin (\beta+3 \theta) \sin \theta d \theta+\lambda_{2} \int_{0}^{\pi} e^{\Gamma} \sin 3 \theta \sin \theta d \theta=0
\end{aligned}
$$

These equations are to be solved together with (27) and (57) for $a_{1}, a_{2}, \lambda_{1}$, and $\lambda_{2}$. The Lagrange multipliers may be eliminated from (57), $(58(1))$, and (58(2)) to give the relation between $a_{1}$ and $a_{2}$ :

$$
\begin{aligned}
f\left(a_{1}, a_{2}\right)=\int_{0}^{\pi} e^{\Gamma} \sin 3 \theta \sin \theta d \theta \int_{0}^{\pi} e^{\Gamma}\left[a_{1} \sin (\beta+\theta)-\cos \beta\right] \sin \theta d \theta \\
\quad-\int_{0}^{\pi} e^{\Gamma} \sin (\beta+3 \theta) \sin \theta d \theta \int_{0}^{\pi} e^{\Gamma}\left[a_{1} \sin \theta-1\right] \sin \theta d \theta=0 .
\end{aligned}
$$

A second relation between $a_{1}$ and $a_{2}$ is given by (27b) with $\kappa$ specified. However, the inverse problem is simpler; in it $a_{1}$ is regarded as known, for then $a_{2}$ is determined by (59), and $\kappa$ is fixed by (27b). Finally, $C_{L}$ may be found from (28), (20) and (16) (with $\alpha=0$ ). 


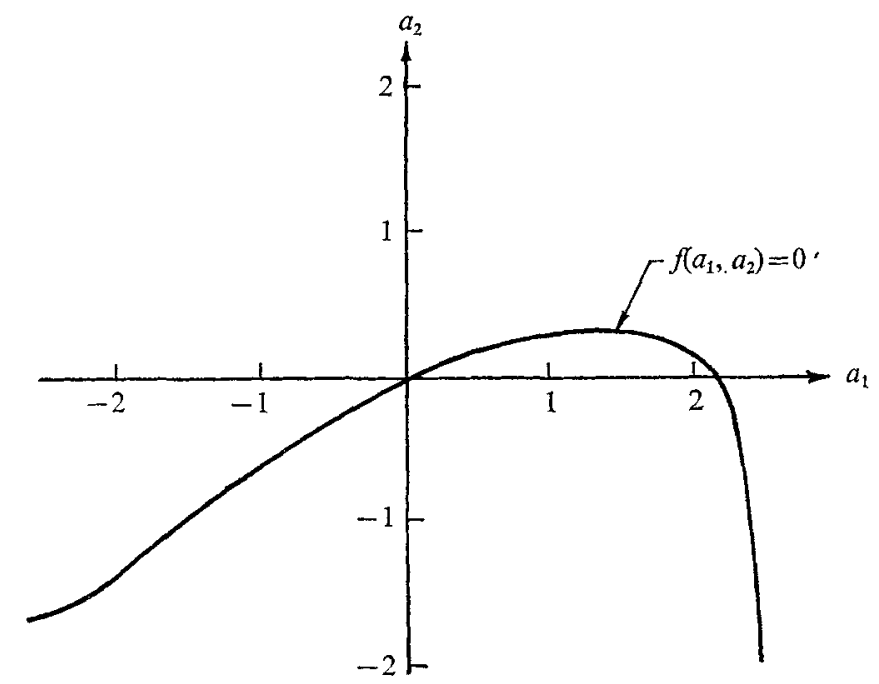

Figure 3. The plot of $f\left(a_{1}, a_{2}\right)=0$ in the two-term Fourier series expansion.

The curve of $a_{2}$ against $a_{1}$ satisfying (59) is shown in figure 3 and the lift coefficient for the two-term Fourier expansion is plotted in figure 2. As $\kappa \rightarrow 0$, it can be shown by expansion of $(27 b),(28)$, and (59) for $\left|a_{1}\right|,\left|a_{2}\right| \ll 1$, that

$$
a_{2}=\frac{7}{17} a_{1}+O\left(a_{1}^{2}\right)
$$

and

$$
C_{L \max }=\frac{\pi}{2}\left(\frac{255}{32}\right)^{\frac{1}{2}} \kappa^{\frac{1}{2}}+O\left(\kappa^{\frac{3}{2}}\right)=4.43 \kappa^{\frac{1}{2}}+O\left(\kappa^{\frac{3}{2}}\right) .
$$

Thus, the maximum lift coefficient increases more slowly (with increasing $\kappa$ ) for the two-term Fourier expansion than for the linearized theory (see (43)). This is thought to be due to the previously mentioned endpoint singularities that are present in the linearized theory.

Actual plate shapes for the case $N=2$ are shown in figure 4 for various values of $\kappa$ (note change of vertical scale). These are found by (15) numerically evaluated for real $\zeta$. The factor $A$ in (15) drops out after normalization of the chord to unity. Note that the maximum height of the plate occurs at two symmetrically located points (at the crosses in figure 4) for smaller values of $\kappa(\lesssim 0.030)$.

The cases $N=3,4, \ldots$, etc., could in theory, be solved as outlined above, and should result in higher and higher lift coefficients for a given ratio of arc-length to chord; however, the calculation difficulties involved in the solution of the system of equations (57) and (58) would surely increase.

\section{Discussion: comparison with the thin-wing theory}

It is of interest to investigate the optimum shape based on the usual thin-wing theory (such as the one adopted by Cumberbatch 1958), now with the gravity effect further neglected). Denoting the planing surface profile by $y=h(x)$, with $\left|h^{\prime}(x)\right| \ll 1$ on $(-1,1)$, and assuming that the velocity $U\left(1+u_{1}, v_{1}\right)$ has only a small perturbation, we have the linearized boundary condition

$$
\begin{aligned}
& v_{1}^{-}(x)=d h / d x \quad(|x|<1), \\
& u_{1}^{-}(x)=0 \quad(|x|>1) .
\end{aligned}
$$




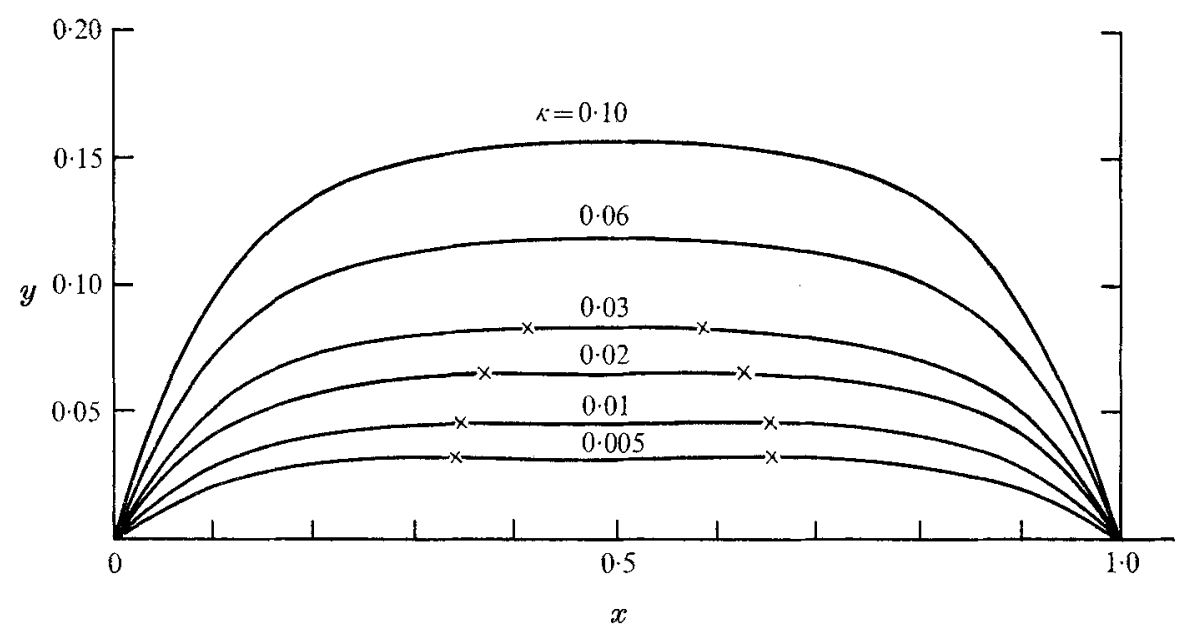

FIGURE 4. Optimum planing surface shapes for the two-term Fourier expansion (crosses denote maximum heights).

A comparison of $(61 a, b)$ with (5)-(11) shows that the perturbation complex velocity $w_{1}=u_{1}-i v_{1}$ in linear theory is exactly analogous to $\omega(\zeta)$ discussed in $\S 2, \omega$ now being approximated by $w_{1}$. In fact, the relationships $(5)-(14)$ will apply if $\omega(\zeta)$ is replacod by $-w_{1}(z)$, and $\zeta=\xi+i \eta$ by $z=x+i y$, and with continuation $w_{1}(\bar{z})=-\overline{w_{1}(z)}$. The lift coefficient provided by the linear theory is

$$
C_{L}=\frac{1}{2} \int_{-1}^{1} C_{p}^{-}(x) d x=-\int_{-1}^{1} u_{1}^{-}(x) d x
$$

For the ratio of wetted arc-length $S$ to chord $l$ we have

$$
\kappa=S / l-1=\frac{1}{2} \int_{-1}^{1}\left(1+h_{x}^{2}\right)^{\frac{1}{2}} d x-1=\frac{1}{4} \int_{-1}^{1}\left[v_{1}^{-}(x)\right]^{2} d x,
$$

upon taking in the last step the Taylor expansion, as is consistent within the linear theory, and using condition (61a).

If we now attempt to minimize the functional

$$
I_{1}\left[u_{1}, v_{1}\right]=\lambda \kappa-C_{L}=\int_{-1}^{1}\left\{\frac{1}{4} \lambda\left[v_{1}^{-}(x)\right]^{2}+u_{1}^{-}(x)\right\} d x,
$$

by the same method as in $\S 2$, now with $u_{1}^{-}$and $v_{1}^{-}$related (by analogy with (11)) by $v_{1}^{-}(x)=-H_{x}\left[u_{1}^{-}\right]$for $|x|<1$, we obtain the Euler equation (by analogy with (26)) as

$$
\frac{\lambda}{2 \pi} \int_{-1}^{1} \frac{v_{1}^{-}(t) d t}{t-x}=-1 \quad(|x|<1)
$$

The solution to $(65)$, having the symmetry $v_{1}^{-}(-x)=-v_{1}^{-}(x)$, integrable at $x= \pm 1$, and corresponding to a $w_{\mathbf{1}}(z)$ that vanishes at infinity, is

$$
v_{1}^{-}(x)=-2 \lambda^{-1} x\left(1-x^{2}\right)^{-\frac{1}{2}} \quad(|x|<1) .
$$

Unfortunately, this $v_{1}^{-}(x)$ is not square-integrable at $x= \pm 1$ to provide a finite $\kappa$ by (63). 
Without further pursuing the optimization calculation on the premises of thinwing theory, we mention below a few solutions by the linear theory to supplement our discussion and comparison with the previous (approximate) optimal solutions. First, of all the 'smooth-entry' planing surfaces that are characterized by the orthogonality condition

$$
\int_{-1}^{1} v_{1}^{-}(x)\left(1-x^{2}\right)^{-\frac{1}{2}} d x=0
$$

a simple example, first given by Cumberbatch (1958), is the parabola

$$
h(x)=\epsilon\left(1-x^{2}\right) \quad(\epsilon \ll 1) .
$$

From this it immediately follows that

and

$$
\begin{gathered}
v_{1}^{ \pm}(x)=-2 \epsilon x, \quad u_{1}^{-}(x)=2 \epsilon\left(1-x^{2}\right)^{\frac{1}{2}}, \quad \kappa=\frac{2}{3} \epsilon^{2}+O\left(\epsilon^{4}\right), \\
C_{L}=\epsilon \pi=\pi(3 \kappa / 2)^{\frac{1}{2}}+O(\kappa) \simeq 3 \cdot 85 \kappa^{\frac{1}{2}}+O(\kappa) .
\end{gathered}
$$

Although this result of linear theory compares quite favourably with (60), derived from a nonlinear calculation of the two-term Fourier expansion, it should be stressed that the linear theory generally over-estimates the lift (for planing surfaces and cavitating hydrofoils).

Another example of linear calculation in close analogy to case (ib) of nearly uniform lift distribution (47) is

$$
u_{1}^{-}=-u_{0}=\text { const. }, \quad v_{1}^{ \pm}(x)=\frac{u_{0}}{\pi} \log \frac{1-x}{1+x} \quad(|x|<1),
$$

corresponding to which we have

$$
\kappa=\frac{1}{4} \int_{-1}^{1}\left[v_{1}^{-}(x)\right]^{2} d x+O\left(u_{0}^{4}\right)=\frac{1}{6} u_{0}^{2}+O\left(u_{0}^{4}\right),
$$

and

$$
C_{L}=2 u_{0}=2(6 \kappa)^{\frac{1}{2}}+O(\kappa)=4 \cdot 9 \kappa^{\frac{1}{2}}+O(\kappa) .
$$

A significant difference between this result and the optimal solution (45) is that case $(\mathrm{i} b)$ is applicable only when $\kappa$ is near a critical upper limit $(\kappa \sim 0 \cdot 1082$, see (45), (46)), and is not for exceedingly small $\kappa$, whereas the present linear theory is supposed to hold for arbitrarily small $\kappa$, in which region the previous solution (43) is superior. The above $C_{L}$, however, is still slightly higher than the $C_{L \max }$ of (60) based on the two-term Fourier expansion; this gain in $C_{L}$ of (70b) may be ascribed to the fact that $v_{1}^{-}(x)$ of $(69)$ now has a weak singularities, rather than being regular at $x= \pm 1$.

This work was carried out under the support of the Naval Ship System Command General Hydrodynamics Research Program, administered by the Naval Ship Research and Development Center and the Office of Naval Research, under contract $220(51)$. 


\section{REFERENCES}

Cumberbatch, E. 1958 Two-dimensional planing at high Froude number. J. Fluid Mech. 4,466 .

Muskhelishvili, N. I. 1953 Singular Integral Equations. Groningen, Holland: Noordhoff.

Rispin, P. P. A. 1967 A singular perturbation method for nonlinear water waves past an obstacle. Ph.D. thesis, California Institute of Technology.

Tricomi, F. G. 1957 Integral Equations. Interscience.

Wehausen, J. V. \& Lattone, E, V. 1960 Surface Waves. Handbuch der Physik, vol. 9. Springer.

WhItNey, A. K. 1969 Minimum drag profiles in infinite cavity flows. Ph.D. thesis, California Institute of Technology.

WV, T. Y. 1967 A singular perturbation theory for nonlinear free-surface flow problems. International Shipbuilding Progress, 14, 88.

WU, T. Y. \& Whitney, A. K. 1971 Theory of optimum shapes in free-surface flows. Part 1. California Institute of Technology Rep. E 132 F. 1. 